\title{
Regulation of Axillary Meristem Initiation by Transcription Factors and Plant Hormones
}

\author{
Minglei Yang and Yuling Jiao* \\ State Key Laboratory of Plant Genomics, Institute of Genetics and Developmental Biology, Chinese Academy of Sciences, \\ and National Center for Plant Gene Research, Beijing, China
}

One distinctive feature of plant post-embryonic development is that plants can undergo reiterative growth and continuous organogenesis throughout their lifetimes. Axillary meristems (AMs) in leaf axils play a central role in this growth and differences in meristem initiation and development produce the diversity of plant architecture. Studies in the past 15 years have shown that several transcription factors (TFs) and phytohormones affect AM initiation. In this review, we highlight recent research using systems biology approaches to examine the regulatory hierarchies underlying AM initiation and the role

OPEN ACCESS

Edited by:

Hirokazu Tsukaya,

The University of Tokyo, Japan

Reviewed by:

Mitsuhiro Aida,

Nara Institute of Science and Technology, Japan

Zhaojun Ding,

Shandong University, China

*Correspondence:

Yuling Jiao

yljiao@genetics.ac.cn

Specialty section: This article was submitted to

Plant Cell Biology,

a section of the journal

Frontiers in Plant Science

Received: 07 October 2015 Accepted: 03 February 2016 Published: 18 February 2016

Citation:

Yang M and Jiao Y (2016) Regulation of Axillary Meristem Initiation by Transcription Factors and Plant Hormones. Front. Plant Sci. 7:183. doi: 10.3389/fpls.2016.00183 of auxins and cytokinins in AM initiation and development. This research revealed a developmental mechanism in which phytohormone signals act with a gene regulatory network containing multiple TFs to contribute to the initiation of AMs.

Keywords: axillary meristem, auxin, cytokinin, transcription factors, post-embryonic development

\section{AXILLARY BUD FORMATION AND LATERAL BRANCHING}

Plant post-embryonic organs originate from meristem tissues, which contain pluripotent cells. During embryonic development, the shoot apical meristem (SAM) and root apical meristem establish the primary axis of the plant and subsequently give rise to shoot and root structures (Pautler et al., 2013). In seed plants, the SAM repeatedly produces phytomers consisting of a leaf, an axillary meristem (AM), and an internode. Each AM functions as a new SAM to establish a secondary growth axis from a lateral bud situated in or near the leaf axils. Thus, SAMs and AMs produce the overall architecture of the plant (Pautler et al., 2013).

Generally, development of axillary buds comprises two stages, initiation in the leaf axil, and subsequent outgrowth or dormancy (Tantikanjana et al., 2001). During vegetative growth in Arabidopsis thaliana, axillary buds initiate at a distance from the SAM and establish an acropetal gradient of axillary bud formation (Hempel and Feldman, 1994). During the reproductive phase, axillary buds initiate near the SAM and form in leaf axils of the youngest primordia where they establish a basipetal pattern of initiation and outgrowth (Hempel and Feldman, 1994). Axillary bud formation seems to undergo differential regulation during vegetative and reproductive phases. AM initiation depends on the leaf axil stem cell niche and establishment of this niche is tightly linked to the formation of the boundary region between the stem and the leaf primordium. In addition, AM initiation is also closely associated with leaf polarity because AMs only develop at the adaxial base of the leaf, facing the SAM (McConnell and Barton, 1998). 
Most mutants with altered patterns of shoot branching, including auxin resistant and more axillary growth mutants in Arabidopsis (Leyser, 1997; Stirnberg et al., 2002), decreased apical dominance mutants in petunia (Petunia hybrida) (Napoli et al., 1999), and ramous mutants in pea (Pisum sativum) (Napoli et al., 1999), exhibit increased axillary bud outgrowth activity. Auxins and strigolactones inhibit axillary bud outgrowth (McSteen and Leyser, 2005; Wang and Li, 2008); by contrast, cytokinins antagonize auxins and strigolactones to promote axillary bud outgrowth (Gomez-Roldan et al., 2008; Janssen et al., 2014). Intriguingly, recent work reported that sugar regulates axillary bud outgrowth, which may explain apical dominance (Mason et al., 2014).

In recent years, a combination of mutant analysis, imaging, and systems biology approaches have been used to understand the process of AM initiation. This review aims to integrate current knowledge about the regulation of AM initiation, especially the role of transcription factors (TFs) and phytohormones.

\section{AXILLARY MERISTEM INITIATION IN Arabidopsis}

AMs initiate during the vegetative growth phase; after the floral transition, floral meristems (FMs) initiate to replace leaves. Analysis of molecular markers indicated that the FM is a specialized AM where the leaf degenerates into a bract or cryptic bract after the floral transition (Long and Barton, 2000). Based on this theory, a lateral organ likely consists of two domains corresponding to the emerging leaf and the AM. These two domains are marked by the expression of the leaf markers AINTEGUMENTA (ANT) and ASYMMETRIC LEAVES 1 (AS1) and the meristem marker SHOOT MERISTEMLESS $(S T M)$, respectively. Rosette leaves, cauline leaves, and FMs, which form during the vegetative, transition to reproductive, and reproductive growth phases, respectively, have different partitions of these two domains (Figures 1A-I) (Grbic, 2005). Whereas the mechanism of bract suppression in Arabidopsis flower has been well studied (Whipple et al., 2010), how the partitions of domains change accompanying phase transition requires further studies.

During vegetative development, each phytomer consists of a large rosette leaf, a short stem segment (internode), and an initially morphologically undetectable AM (McSteen and Leyser, 2005). AMs initiate at the junction between the stem and the adaxial base of the subtending leaf. Cells in leaf axils are smaller in volume than their neighbors and are presumably meristematic at this stage. The expression of STM can be detected in leaf axils of all stages (Figure 1J) (Grbic and Bleecker, 2000; Long and Barton, 2000; Greb et al., 2003), although it remains

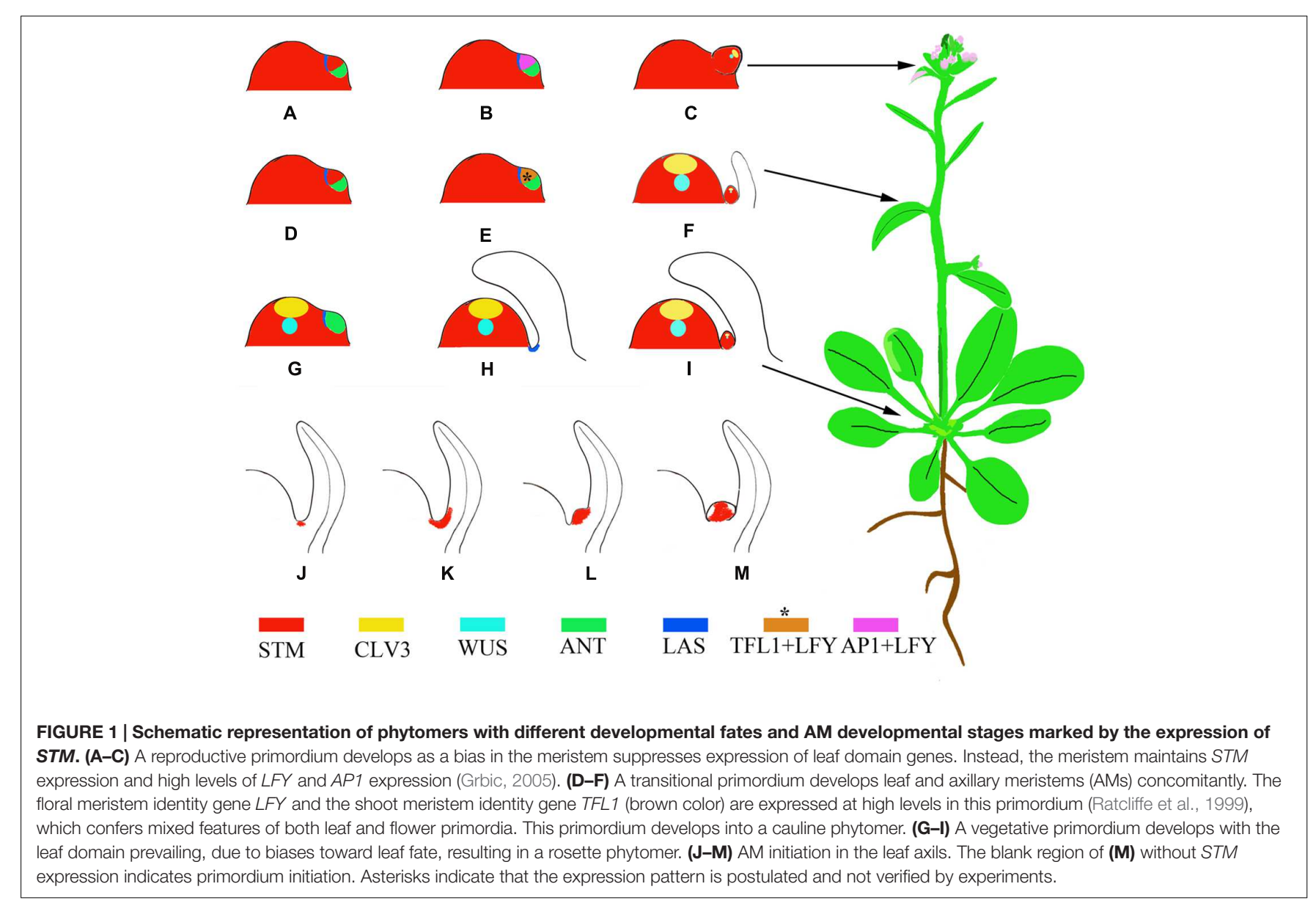


to be tested if a cell population that continuously expresses STM exists. Because the STM-expressing cells 'move' upward toward the leaf, the initial STM-expressing cells may create a separation while their neighboring cells re-differentiate as AM progenitor cells (Long and Barton, 2000). The STM-expressing meristematic cells decrease in number during leaf growth and finally become restricted to the center of the leaf axil. The first visible change in the leaf axil is the appearance of a group of dense-staining, fast-dividing cells surrounded by the beneath shell zone cells (Shah and Patel, 1972; Grbic and Bleecker, 2000; Wang et al., 2014b), a well-developed, curved zone of elongated, cambiform, and vacuolated cells that delimits the meristematic cells from the adjacent tissue. Prior to axillary bud formation, STM expression is up-regulated in meristematic cells (Figure 1K) (Long and Barton, 2000; Greb et al., 2003). Subsequent division of the meristematic cells in the leaf axil leads to the morphologically distinguishable bump on the adaxial leaf base (Figure 1L) and eventually a visible axillary bud forms having its own leaf primordia (Figure 1M).

Following the floral transition of the SAM, the two or three leaf primordia formed just prior to the transition develop into cauline leaves. The cauline leaves are smaller than rosette leaves and are associated with actively growing axillary buds (Hempel and Feldman, 1994). The AMs subtended by cauline leaves develop concomitantly with leaves (Grbic, 2005). Both TERMINAL FLOWER1 (TFL1), which inhibits FM fate, and LEAFY (LFY), which promotes FM fate, are expressed during this period (Ratcliffe et al., 1998; Grbic, 2005) (Figures 1D-F).

After the floral transition, the SAM is termed an inflorescence meristem and forms primordia with floral fates. Each primordium develops into an FM subtended by a cryptic bract and has an elongated internode. The morphologically invisible cryptic bract can be detected as a region that expresses ANT but not STM and is considered to have similarities to a leaf primordium. In the primordium, ANT expression ceases at an early stage, leading to a failure of bract growth (Long and Barton, 2000).

Von Goethe proposed that a flower can be considered a compressed shoot (Dornelas and Dornelas, 2005). However, a flower differs from a shoot in its determinate growth, which results from transient AM activity and produces a limited number of floral organs. In addition, a flower does not branch because its floral organs, like sepals, do not have AMs. APETALA1 (AP1) and homologous MADS-family TF genes mediate the suppression of sepal axil AM formation and mutations of these genes result in secondary flowers in sepal axils (Irish and Sussex, 1990; Mandel et al., 1992; Sundstrom et al., 2006). The regulation of secondary FMs of a sepal axil shares similarity with the regulation of vegetative AMs (Han et al., 2014).

\section{TRANSCRIPTION FACTORS IN AXILLARY MERISTEM INITIATION}

Most of the known genes that affect AM initiation encode TFs. AMs initiate in the leaf axils, closely associated with the boundary zone that separates the leaf primordium from the shoot meristem. Many of the genes that affect AM initiation also influence boundary formation (Wang et al., 2016), although genes affecting boundary formation do not necessarily affect AM initiation.

LATERAL SUPPRESSOR (LAS) encodes a member of the GRAS family of TFs (Greb et al., 2003), and Arabidopsis las mutants have vegetative stage-specific defects in AM initiation. LAS homologs also affect reproductive stage development in tomato (Solanum lycopersicum) and rice (Oryza sativa) (Groot et al., 1994; Schumacher et al., 1999; Li et al., 2003). LAS transcripts specifically accumulate in the axils of primordia, which derive from the SAM during vegetative and reproductive development (Greb et al., 2003).

REVOLUTA $(R E V)$ encodes a class III homeodomain/leucine zipper TF (HD-ZIPIII). Unlike las mutants, rev mutants show reduced AM formation in both vegetative and reproductive stages (Otsuga et al., 2001). In addition, $R E V$ has a broad expression pattern in many tissue types in shoot and root. In addition to AM initiation, rev mutants show defects in the development of the SAM, leaves, vasculature, and roots.

Members of the redundant family of REGULATOR OF AXILLARY MERISTEMS (RAX) genes encode Myb-like TFs and affect AM initiation in Arabidopsis and in tomato (Keller et al., 2006). Compared to $L A S$, expression of $R A X 1$ is spatially more restricted at the center of the leaf axil and $R A X 1$ is the earliest known marker of the position of the AM (Keller et al., 2006).

In addition, NAC domain proteins CUC1, CUC2, and CUC3 have redundant but partially distinct functions in boundary formation and AM initiation. The CUC genes have boundary-specific expression, but different functions. CUC1 shows no obvious role in AM initiation, CUC2 slightly affects AM initiation, and CUC3 functions as a major regulator of AM initiation (Hibara et al., 2006). Compared with the single mutants, the las cuc3 double mutants exhibit more frequent axillary bud defects, suggesting that LAS and CUC3 have overlapping functions in AM initiation.

Genetic studies and genome-scale analyses have begun to uncover the gene regulatory network (GRN) underlying AM initiation. For example, CUC genes regulate LAS expression (Hibara et al., 2006; Raman et al., 2008) and CUC proteins directly bind to the LAS promoter and activate its expression (Tian et al., 2014). Cell type-specific gene expression analysis and genome-scale yeast-one-hybrid assays showed that CUC2 and LAS function as two hubs of this GRN, with their promoter regions connected to many TFs (Tian et al., 2014). For example, $R A X 1$ and 3 directly activate CUC2; CUC2 then directly activates the expression of the miRNA gene MIR164c and miR164 degrades CUC1 and 2 transcripts to form a regulatory loop (Laufs et al., 2004; Mallory et al., 2004; Larue et al., 2009; Tian et al., 2014). GRN analyses have also identified new regulators of AM initiation. For example, DORNRÖSCHEN regulates AM initiation through direct activation of CUC2 (Tian et al., 2014). SQUAMOSA-PROMOTER BINDING PROTEINLIKE proteins suppress AM initiation by directly repressing LAS expression (Figure 2) and thus connect the aging pathway to AM initiation. 


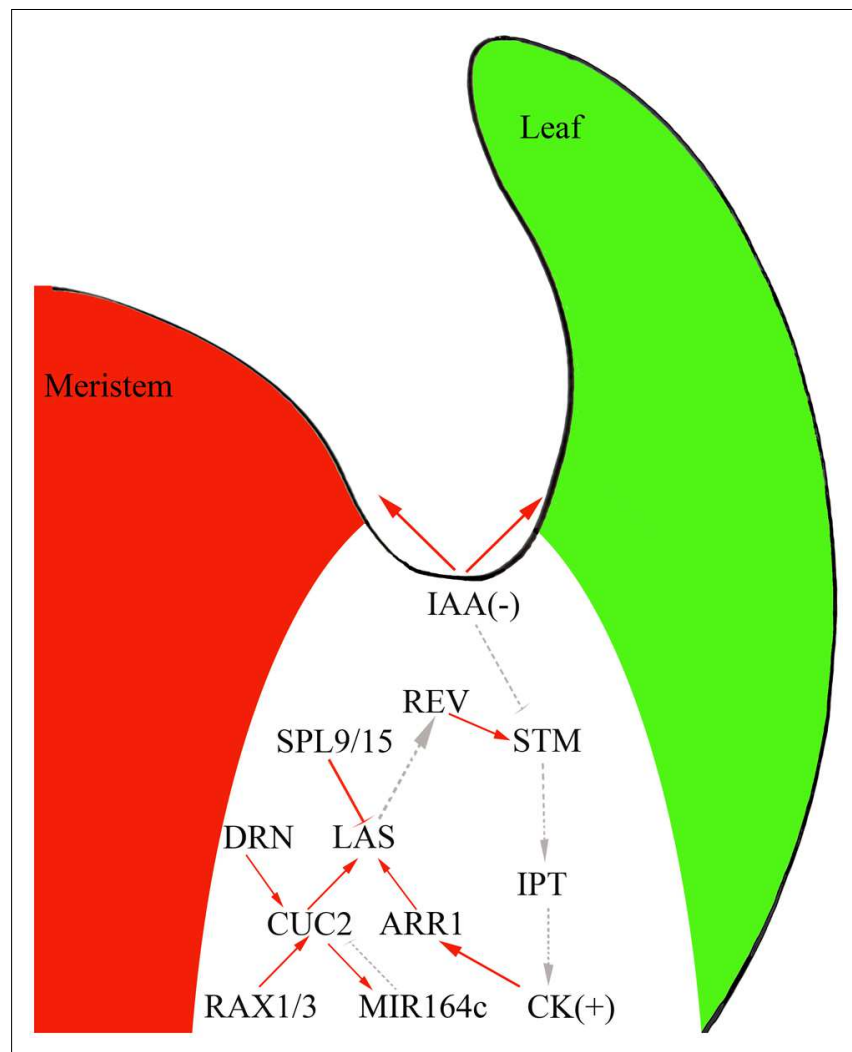

FIGURE 2 | The interplay of phytohormones and transcription factors (TFs) to control AM initiation in the boundary domain. Red solid line, direct interaction; gray dotted line, genetic interaction; arrow, activation effect; bar, repressive effect.

\section{PHYTOHORMONES IN AXILLARY MERISTEM INITIATION}

Auxin and cytokinins have broad and vital effects on plant development and recent studies have suggested that these phytohormones also regulation $\mathrm{AM}$ initiation. Indeed, experiments using the DII-Venus auxin sensor (Vernoux et al., 2011) showed that the leaf axil region where AMs initiate has low auxin concentrations (Wang et al., 2014a,b). AM initiation requires this auxin minimum; ectopic production of the auxin biosynthesis enzyme iaaM in the leaf axil can lead to compromised AM initiation. In leaf axils, the expression of the meristematic cell marker STM decreases, but the expression of LAS remains unchanged (Wang et al., 2014b). The leaf axil auxin minimum depends on auxin efflux and influx. In pin-formed1 mutants, which affect a key auxin intercellular efflux transporter, and aux1 mutants, which show compromised auxin influx, the auxin minimum in the leaf axil disappears and AM initiation fails to take place (Wang et al., 2014a,b).

Following the formation of the auxin minimum, cytokinin accumulates at the leaf axil (Wang et al., 2014b) as detected by the synthetic reporter pTCS:GFP-ER (Muller and Sheen, 2008). The leaf axil cytokinin signaling pulse depends on the earlier auxin minimum. Normal AM initiation requires cytokinin biosynthesis and signaling, as mutants of cytokinin biosynthetic genes (Müller et al., 2015), cytokinin receptor genes, and downstream B-type ARABIDOPSIS RESPONSE REGULATOR $(B-A R R)$ TF genes (Wang et al., 2014b) compromise AM initiation. Also, STM can activate IPT expression to promote cytokinin biosynthesis (Jasinski et al., 2005; Yanai et al., 2005).

The existence of a linear regulatory pathway can be speculated, where the auxin minimum leads to STM expression, which enhances cytokinin biosynthesis and signaling. As cytokinins can act upstream of STM (Rupp et al., 1999), cytokinin and STM could establish a positive feedback loop during AM initiation. Furthermore, AM initiation also involves morecomplicated regulatory interactions between phytohormones and TFs. For instance, exogenous cytokinin can restore AM initiation in rax mutants (Wang et al., 2014b). Also, ARR1, a TF downstream of cytokinin signaling, binds to the LAS promoter to promote its expression (Tian et al., 2014).

In addition to auxin and cytokinins, the leaf axil GRN also identified additional phytohormone signals that affect AM initiation (Tian et al., 2014). One candidate of particular interest is brassinosteroids (BRs). Recent findings have shown that BRs regulate organ boundary-specific gene expression and contribute to boundary formation (Bell et al., 2012; Gendron et al., 2012). Within the boundary zone, $L O B$ can directly promote the expression of PHYB ACTIVATION TAGGED SUPPRESSOR1 (BAS1), which encodes a BR-inactivating enzyme (Bell et al., 2012). Consistent with this, ectopic expression of $L O B$ leads to a reduced BR response. Also, $L O B$ dysfunction results in organ fusion, which can be suppressed by BAS1 expression under the $L O B$ promoter, indicating that BR hyperaccumulation contributes to boundary establishment in lob mutants (Bell et al., 2012). One hypothesis proposes that a local burst of BR signaling leads to activation of $L O B$ expression, which in turn inactivates BR signaling (Arnaud and Laufs, 2013).

Of particular interest is the observation that the BR-activated gene BRASSINAZOLE-RESISTANT1 (BZR1) represses CUC3 via BZR1 directly binding to the CUC3 promoter (Gendron et al., 2012). CUC2 and CUC3 positively regulate $L O F 1$ expression in the boundary zone (Gendron et al., 2012). Thus, BZR1 indirectly causes the reduced expression of $L O F 1$, possibly by repression of CUC3. Hence, the BR content decreases due to the $L O B$ dependent activation of $B A S 1$, which results in a decrease of $B Z R 1$ and an increase of CUC and LOF1 levels in the boundary region (Bell et al., 2012; Gendron et al., 2012). Whether BR and its signaling affect AM initiation remains to be tested.

\section{CONCLUSION AND PERSPECTIVES}

Recent efforts to understand AM initiation have showed that various TFs and phytohormones collaboratively orchestrate initiation of AMs. However, many open questions still require further investigation. Like cells of the stomatal lineage, AM 
progenitor cells may represent a meristematic cell lineage. It will be important to understand the major shifts accompanying each fate transition from the designation of meristematic cells, to the maintenance of meristematic cell fate, and finally the activation of these cells to form new buds. For example, future work will address whether transcriptional regulation works in coordination with epigenetic mechanisms to affect cell fate determination during AM initiation. We also need to identify more regulators of AM initiation to obtain a comprehensive picture of this regulatory pathway.

\section{AUTHOR CONTRIBUTIONS}

ML and YJ wrote the manuscript.

\section{REFERENCES}

Arnaud, N., and Laufs, P. (2013). Plant development: brassinosteroids go out of bounds. Curr. Biol. 23, R152-R154. doi: 10.1016/j.cub.2013.01.001

Bell, E. M., Lin, W. C., Husbands, A. Y., Yu, L., Jaganatha, V., Jablonska, B., et al. (2012). Arabidopsis lateral organ boundaries negatively regulates brassinosteroid accumulation to limit growth in organ boundaries. Proc. Natl. Acad. Sci. U.S.A. 109, 21146-21151. doi: 10.1073/pnas.1210789109

Dornelas, M. C., and Dornelas, O. (2005). From leaf to flower: revisiting Goethe’s concepts on the "metamorphosis" of plants. Braz. J. Plant Physiol. 17, 335-343. doi: 10.1590/S1677-04202005000400001

Gendron, J. M., Liu, J. S., Fan, M., Bai, M. Y., Wenkel, S., Springer, P. S., et al. (2012). Brassinosteroids regulate organ boundary formation in the shoot apical meristem of Arabidopsis. Proc. Natl. Acad. Sci. U.S.A. 109, 21152-21157. doi: 10.1073/pnas.1210799110

Gomez-Roldan, V., Fermas, S., Brewer, P. B., Puech-Pages, V., Dun, E. A., Pillot, J.-P., et al. (2008). Strigolactone inhibition of shoot branching. Nature 455, 189-194. doi: 10.1038/nature07271

Grbic, V. (2005). Comparative analysis of axillary and floral meristem development. Can. J. Bot. 83, 343-349. doi: 10.1139/b05-017

Grbic, V., and Bleecker, A. B. (2000). Axillary meristem development in Arabidopsis thaliana. Plant J. 21, 215-223. doi: 10.1046/j.1365-313x.2000.00670.x

Greb, T., Clarenz, O., Schafer, E., Muller, D., Herrero, R., Schmitz, G., et al. (2003). Molecular analysis of the Lateral Suppressor gene in Arabidopsis reveals a conserved control mechanism for axillary meristem formation. Genes Dev. 17, 1175-1187. doi: 10.1101/gad.260703

Groot, S. P. C., Keizer, L. C. P., Ruiter, W. D., and Dons, J. J. M. (1994). Seed and fruit set of the lateral suppressor mutant of tomato. Sci. Hortic. 59, 157-162. doi: 10.1016/0304-4238(94)90082-5

Han, Y., Zhang, C., Yang, H., and Jiao, Y. (2014). Cytokinin pathway mediates APETALA1 function in the establishment of determinate floral meristems in Arabidopsis. Proc. Natl. Acad. Sci. U.S.A. 111, 6840-6845. doi: 10.1073/pnas.1318532111

Hempel, F., and Feldman, L. (1994). Bi-directional inflorescence development in Arabidopsis thaliana: acropetal initiation of flowers and basipetal initiation of paraclades. Planta 192, 276-286. doi: 10.1007/BF01089045

Hibara, K., Karim, M. R., Takada, S., Taoka, K., Furutani, M., Aida, M., et al. (2006). Arabidopsis CUP-SHAPED COTYLEDON3 regulates postembryonic shoot meristem and organ boundary formation. Plant Cell 18, 2946-2957. doi: $10.1105 /$ tpc. 106.045716

Irish, V. F., and Sussex, I. M. (1990). Function of the apetala-1 gene during Arabidopsis floral development. Plant Cell 2, 741-753. doi: 10.1105/tpc.2.8.741

Janssen, B. J., Drummond, R. S., and Snowden, K. C. (2014). Regulation of axillary shoot development. Curr. Opin. Plant Biol. 17, 28-35. doi: 10.1016/j.pbi.2013.11.004

Jasinski, S., Piazza, P., Craft, J., Hay, A., Woolley, L., Rieu, I., et al. (2005). KNOX action in Arabidopsis is mediated by coordinate regulation of cytokinin

\section{FUNDING}

The authors thank Muhammad Sajjad for proofreading. The authors' laboratory is supported by the National Basic Research Program of China (973 Program) Grant 2014CB943500, National Natural Science Foundation of China grants 31430010 and 31222033, the National Program for Support of Top-Notch Young Professionals, and State Key Laboratory of Plant Genomics grant SKLPG2011B0103.

\section{ACKNOWLEDGMENTS}

YJ thanks Ottoline Leyser, Jiayang Li, and Klaus Theres for stimulating discussions. We apologize to those authors whose work could not be cited due to space limitations.

and gibberellin activities. Curr. Biol. 15, 1560-1565. doi: 10.1016/j.cub.2005. 07.023

Keller, T., Abbott, J., Moritz, T., and Doerner, P. (2006). Arabidopsis Regulator Of Axillary Meristems 1 controls a leaf axil stem cell niche and modulates vegetative development. Plant Cell 18, 598-611. doi: 10.1105/tpc.105.038588

Larue, C. T., Wen, J., and Walker, J. C. (2009). A microRNA-transcription factor module regulates lateral organ size and patterning in Arabidopsis. Plant J. 58, 450-463. doi: 10.1111/j.1365-313X.2009.03796.x

Laufs, P., Peaucelle, A., Morin, H., and Traas, J. (2004). MicroRNA regulation of the CUC genes is required for boundary size control in Arabidopsis meristems. Development 131, 4311-4322. doi: 10.1242/dev.01320

Leyser, O. (1997). Auxin: lessons from a mutant weed. Physiol. Plant. 100, 407-414. doi: 10.1111/j.1399-3054.1997.tb03044.x

Li, X., Qian, Q., Fu, Z., Wang, Y., Xiong, G., Zeng, D., et al. (2003). Control of tillering in rice. Nature 422, 618-621. doi: 10.1038/nature01518

Long, J., and Barton, M. K. (2000). Initiation of axillary and floral meristems in Arabidopsis. Dev. Biol. 218, 341-353. doi: 10.1006/dbio.1999.9572

Mallory, A. C., Dugas, D. V., Bartel, D. P., and Bartel, B. (2004). MicroRNA regulation of NAC-domain targets is required for proper formation and separation of adjacent embryonic, vegetative, and floral organs. Curr. Biol. 14, 1035-1046. doi: 10.1016/j.cub.2004.06.022

Mandel, M. A., Gustafson-Brown, C., Savidge, B., and Yanofsky, M. F. (1992). Molecular characterization of the Arabidopsis floral homeotic gene APETALA1. Nature 360, 273-277. doi: 10.1038/360273a0

Mason, M. G., Ross, J. J., Babst, B. A., Wienclaw, B. N., and Beveridge, C. A. (2014). Sugar demand, not auxin, is the initial regulator of apical dominance. Proc. Natl. Acad. Sci. U.S.A. 111, 6092-6097. doi: 10.1073/pnas.1322045111

McConnell, J. R., and Barton, M. K. (1998). Leaf polarity and meristem formation in Arabidopsis. Development 125, 2935-2942.

McSteen, P., and Leyser, O. (2005). Shoot branching. Annu. Rev. Plant Biol. 56, 353-374. doi: 10.1146/annurev.arplant.56.032604.144122

Muller, B., and Sheen, J. (2008). Cytokinin and auxin interaction in root stemcell specification during early embryogenesis. Nature 453, 1094-1097. doi: 10.1038/nature06943

Müller, D., Waldie, T., Miyawaki, K., To, J. P. C., Melnyk, C. W., Kieber, J. J., et al. (2015). Cytokinin is required for escape but not release from auxin mediated apical dominance. Plant J. 82, 874-886. doi: 10.1111/tpj.12862

Napoli, C. A., Beveridge, C. A., and Snowden, K. C. (1999). Reevaluating concepts of apical dominance and the control of axillary bud outgrowth. Curr. Top. Dev. Biol. 44, 127-169. doi: 10.1016/S0070-2153(08)60469-X

Otsuga, D., Deguzman, B., Prigge, M. J., Drews, G. N., and Clark, S. E. (2001). Revoluta regulates meristem initiation at lateral positions. Plant J. 25, 223-236. doi: 10.1046/j.1365-313x.2001.00959.x

Pautler, M., Tanaka, W., Hirano, H. Y., and Jackson, D. (2013). Grass meristems I: shoot apical meristem maintenance, axillary meristem determinacy and the floral transition. Plant Cell Physiol. 54, 302-312. doi: 10.1093/pcp/ pct025 
Raman, S., Greb, T., Peaucelle, A., Blein, T., Laufs, P., and Theres, K. (2008). Interplay of miR164, CUP-SHAPED COTYLEDON genes and Lateral Suppressor controls axillary meristem formation in Arabidopsis thaliana. Plant J. 55, 65-76. doi: 10.1111/j.1365-313X.2008.03483.x

Ratcliffe, O. J., Amaya, I., Vincent, C. A., Rothstein, S., Carpenter, R., Coen, E. S., et al. (1998). A common mechanism controls the life cycle and architecture of plants. Development 125, 1609-1615.

Ratcliffe, O. J., Bradley, D. J., and Coen, E. S. (1999). Separation of shoot and floral identity in Arabidopsis. Development 126, 1109-1120.

Rupp, H. M., Frank, M., Werner, T., Strnad, M., and Schmulling, T. (1999). Increased steady state mRNA levels of the STM and KNAT1 homeobox genes in cytokinin overproducing Arabidopsis thaliana indicate a role for cytokinins in the shoot apical meristem. Plant J. 18, 557-563. doi: 10.1046/j.1365313X.1999.00472.x

Schumacher, K., Schmitt, T., Rossberg, M., Schmitz, G., and Theres, K. (1999). The Lateral suppressor (Ls) gene of tomato encodes a new member of the VHIID protein family. Proc. Natl. Acad. Sci. U.S.A. 96, 290-295. doi: 10.1073/pnas.96.1.290

Shah, J. J., and Patel, J. D. (1972). The shell zone: its differentiation and probable function in some dicotyledons. Am. J. Bot. 59, 683-690. doi: 10.2307/2441139

Stirnberg, P., Van De Sande, K., and Leyser, H. M. (2002). MAX1 and MAX2 control shoot lateral branching in Arabidopsis. Development 129, 1131-1141.

Sundstrom, J. F., Nakayama, N., Glimelius, K., and Irish, V. F. (2006). Direct regulation of the floral homeotic APETALA1 gene by APETALA3 and PISTILLATA in Arabidopsis. Plant J. 46, 593-600. doi: 10.1111/j.1365313X.2006.02720.x

Tantikanjana, T., Yong, J. W., Letham, D. S., Griffith, M., Hussain, M., Ljung, K., et al. (2001). Control of axillary bud initiation and shoot architecture in Arabidopsis through the SUPERSHOOT gene. Genes Dev. 15, 1577-1588. doi: $10.1101 /$ gad.887301

Tian, C., Zhang, X., He, J., Yu, H., Wang, Y., Shi, B., et al. (2014). An organ boundary-enriched gene regulatory network uncovers regulatory hierarchies underlying axillary meristem initiation. Mol. Syst. Biol. 10, 755. doi: $10.15252 / \mathrm{msb} .20145470$
Vernoux, T., Brunoud, G., Farcot, E., Morin, V., Van Den Daele, H., Legrand, J., et al. (2011). The auxin signalling network translates dynamic input into robust patterning at the shoot apex. Mol. Syst. Biol. 7, 508. doi: 10.1038/msb.2011.39

Wang, Q., Hasson, A., Rossmann, S., and Theres, K. (2016). Divide et impera: boundaries shape the plant body and initiate new meristems. New Phytol. 209, 485-498. doi: 10.1111/nph.13641

Wang, Q., Kohlen, W., Rossmann, S., Vernoux, T., and Theres, K. (2014a). Auxin depletion from the leaf axil conditions competence for axillary meristem formation in Arabidopsis and tomato. Plant Cell 26, 2068-2079. doi: 10.1105/tpc.114.123059

Wang, Y., Wang, J., Shi, B., Yu, T., Qi, J., Meyerowitz, E. M., et al. (2014b). The stem cell niche in leaf axils is established by auxin and cytokinin in Arabidopsis. Plant Cell 26, 2055-2067. doi: 10.1105/tpc.114.123083

Wang, Y., and Li, J. (2008). Molecular basis of plant architecture. Annu. Rev. Plant Biol. 59, 253-279. doi: 10.1146/annurev.arplant.59.032607. 092902

Whipple, C. J., Hall, D. H., DeBlasio, S., Taguchi-Shiobara, F., Schmidt, R. J., and Jackson, D. P. (2010). A conserved mechanism of bract suppression in the grass family. Plant Cell 22, 565-578. doi: 10.1105/tpc.109. 073536

Yanai, O., Shani, E., Dolezal, K., Tarkowski, P., Sablowski, R., Sandberg, G., et al. (2005). Arabidopsis KNOXI proteins activate cytokinin biosynthesis. Curr. Biol. 15, 1566-1571. doi: 10.1016/j.cub.2005.07.060

Conflict of Interest Statement: The authors declare that the research was conducted in the absence of any commercial or financial relationships that could be construed as a potential conflict of interest.

Copyright (c) 2016 Yang and Jiao. This is an open-access article distributed under the terms of the Creative Commons Attribution License (CC BY). The use, distribution or reproduction in other forums is permitted, provided the original author(s) or licensor are credited and that the original publication in this journal is cited, in accordance with accepted academic practice. No use, distribution or reproduction is permitted which does not comply with these terms. 